\title{
BRAIN CHANGES IN RUPTURED INTRACRANIAL ANEURYSM
}

\author{
BY \\ B. E. TOMLINSON \\ From the Department of Pathology, Newcastle General Hospital
}

(RECEIVED FOR PUbLICATION FEBRUARY 19, 1959)

Since the papers of Beadles (1907), Fearnsides (1916), and Symonds (1924), there have been numerous publications on the clinical manifestations, prognosis, and treatment of spontaneous subarachnoid haemorrhage. The pathology of the brain has received less attention. Many accounts have been taken from routine necropsy reports and few have included detailed examinations of the brain. Intracerebral bleeding has received most attention, largely since the paper of Richardson and Hyland (1941), but recently ischaemic lesions have been more widely recognized (Robertson, 1949; Wilson, Riggs, and Rupp, 1954; Bebin and Currier, 1957). Robertson (1949) described five instances of ischaemia, and it is noteworthy that three were seen in the 10 cases he observed himself, whereas only two were recorded in more than 80 routine post-mortem reports he reviewed. The greatest incidence of infarction is reported by Wilson et al. (1954), who noted focal ischaemic lesions in $45 \%$ of their necropsy material. No account, to my knowledge, however, gives details of the distribution, size, and importance of ischaemic lesions and their relation to other findings in the brain.

This paper reports the brain findings in a series of 32 consecutive cases of fatal ruptured intracranial aneurysms. Two further instances have been excluded because surgical intervention made assessment of the pre-operative findings impossible. The ischaemic lesions found are described in detail, as are the instances of localized subarachnoid collections of blood (subarachnoid haematomata) which may well be important in the production of ischaemic lesions and intracerebral haemorrhage. Mention is also made of granular cell degeneration of the cerebellum which is uncommon and probably a terminal event.

\section{Method of Investigation}

At necropsy the brain was examined externally only, and the bleeding aneurysm located where this was possible without disturbing the blood lying in the fissures, or between the lobes. After fixation for one to two months in formol-saline, the basal vessels were removed from the specimen. The brain was then cut into numerous slices and the distribution of blood in the sulci and fissures noted; material for histology was taken from many areas of the cerebral cortex, basal ganglia, brain-stem, and cerebellum. Particular attention was paid to any area suggestive of infarction and sufficient sections were taken to map out the area involved.

Haematoxylin and eosin staining was used routinely, but Nissl, Mallory's phosphotungstic-acidhaematoxylin, Holzer, myelin, neurofibrillary, and fat stains were used where needed.

\section{Results}

Multiple aneurysms were found in 14 cases; in one of these, many small aneurysms were present on an angiomatous malformation in the Sylvian fissure. In a further case, a single aneurysm on an anterior cerebral artery was associated with an angioma in the frontal lobe on the same side. Thus, in 15 out of 32 cases (over $46 \%$ ), multiple possible sources of bleeding existed. This is a higher percentage of multiple aneurysms than usually reported. Robertson (1949) found 5\%, Bigelow (1955) 10\%, Wilson et al. (1954) $19 \%$, and Richardson and Hyland (1941) $25 \%$. The true figure may not be as high as in this short series, but it will be far higher than is usually reported. Small aneurysms arising on the superior aspect of the circle of Willis will only be detected after the vessels have been dissected off the brain.

The ruptured aneurysms were, in all but one instance, situated anteriorly on the circle of Willis or its branches. Commonly, two vessels were 
involved by the aneurysm arising at a bifurcation, a fact of surgical importance and one which may influence the distribution of the ischaemic lesions. Subdural haematomas were seen twice from ruptured internal carotid aneurysms, but on neither occasion were they large enough to be of practical importance. Some surface subarachnoid haemorrhage was visible in all but one case, and, in this, separation of the frontal lobes revealed a massive haemorrhage. In 14 the surface bleeding was slight, but in 17 it was generalized, and at least $2-3 \mathrm{~mm}$. thick over some portion of the brain. In only three of the 32 cases was massive generalized subarachnoid bleeding the only significant finding. The other $29(90 \%)$ had additional lesions.

\section{Localized Collections of Blood in Subarachnoid Space (Subarachnoid Haematomata). - The} impression that, at most, a little blood clot is met with in the subarachnoid space (Falconer, 1954 ) is shown to be incorrect by the examination of brains which have been fixed without disturbance of the sulci or fissures. With or without generalized subarachnoid bleeding, sulci close to the aneurysm are often widened by blood. This may spread in the depth of the sulci

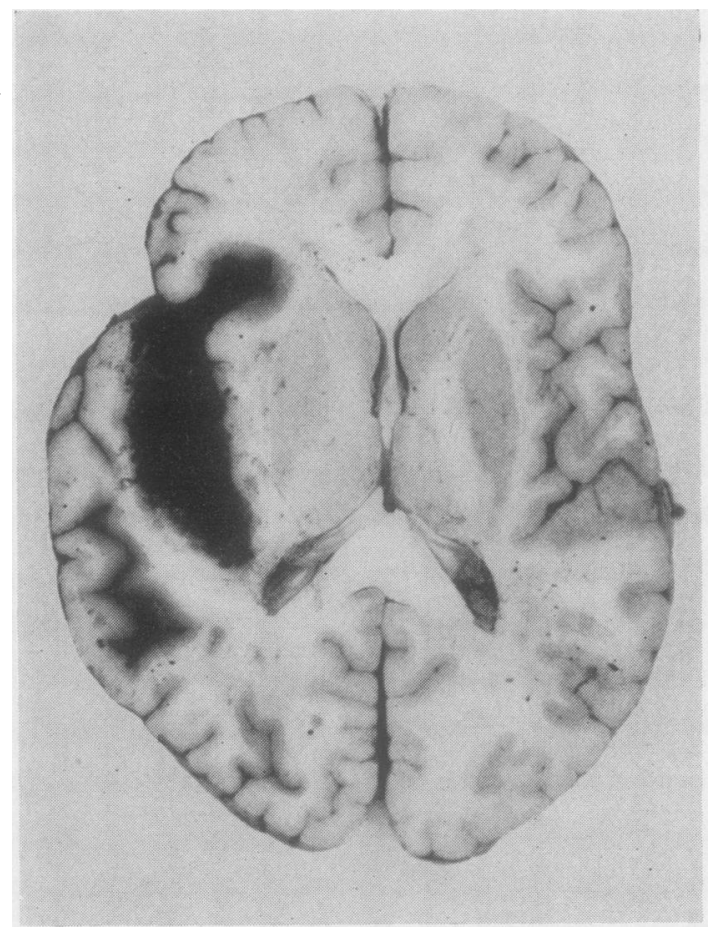

Fig. 1.-A massive collection of blood confined to the Sylvian fissure and depths of neighbouring sulci.

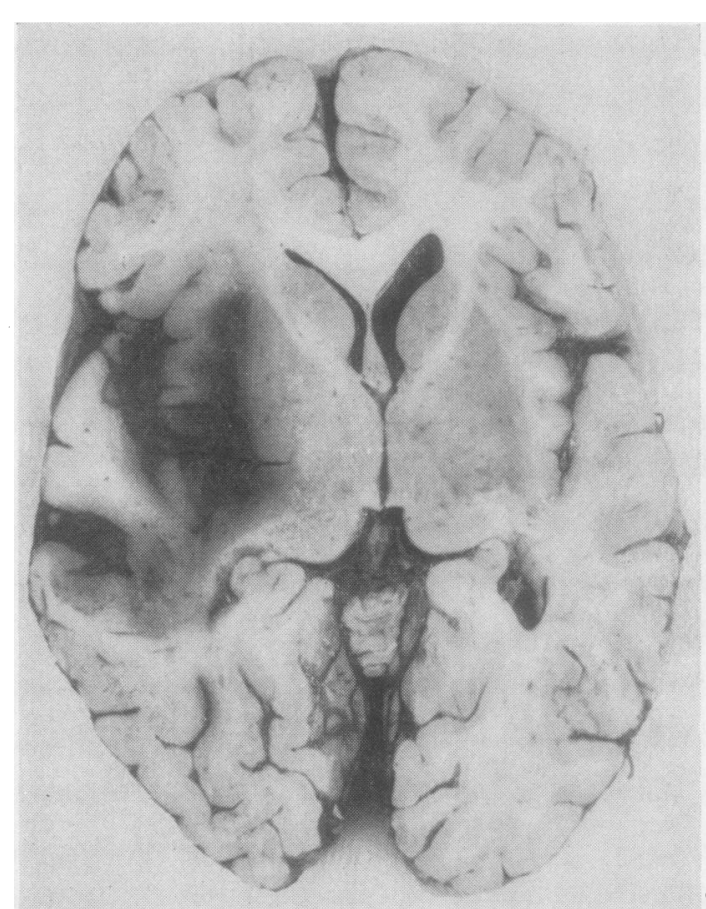

Fig. 2.-Blood in the Sylvian fissure with rupture down a sulcus onthe insular cortex. Infarction surrounding distended neighbour ing sulcus.

for considerable distances and be from 0.5 to 1.5 $\mathrm{cm}$. thick and accompanied by infarction in the surrounding cortex. Subarachnoid haematomata are particularly liable to collect between the frontal lobes from ruptured aneurysms on the anterior communicating and anterior cerebral arteries, and in the Sylvian fissures from ruptured middle cerebral aneurysms. In these situations, the blood may be confined to the subarachnoid space, or may rupture into the cerebral tissue, whilst little escapes on to the brain surface. Large haematomata act as space-occupying masses, and are not infrequently associated with infarction.

In this series, large haematomata, totally or partially confined to the subarachnoid space, were present in nine $(28 \%)$ brains.

Three very large collections were seen in the Sylvian fissure from ruptured middle cerebral aneurysms, one (Fig. 1) being entirely confined to the fissure and causing death by acting as a space-occupying lesion. The white matter of the temporal lobe was markedly oedematous and foci of acute neuronal degeneration were present in the insular cortex. In the second instance (Fig. 2) a massive clot was present in the Sylvian fissure 
and had caused limited intracerebral bleeding by breaching the deepest limit of an insular sulcus. The blood also greatly widened a neighbouring sulcus. The insular and much of the temporal cortex was infarcted. Again, little surface bleeding was present. In the third case a mass of blood, $6 \mathrm{~cm}$. long and $3 \mathrm{~cm}$. wide, in the Sylvian fissure had ruptured into the postero-superior aspect of the insula, producing intracerebral and some intraventricular bleeding; pontine and peduncular haemorrhages, the result of the supratentorial mass, were present. The aneurysm was partly embedded in cerebral tissue but had not ruptured directly into the brain, the breach of the insular cortex being far posterior to the aneurysm. Small areas of acute infarction were present in the cortex of the middle and superior temporal gyri.

Six large subarachnoid haematomata. were associated with ruptured aneurysms on the anterior cerebral or anterior communicating arteries. From these sites the blood may burrow into the sulci on the medial and inferior surfaces of the frontal lobes, or collect between the frontal lobes. If this occurs the thin rostrum of the corpus callosum may rupture with resulting intraventricular bleeding, as seen in Fig. 3. In this patient subarachnoid bleeding was present long before the intraventricular haemorrhage, since, three weeks before death, the patient had recurrent headaches with blood-stained cerebrospinal fluid, before becoming deeply

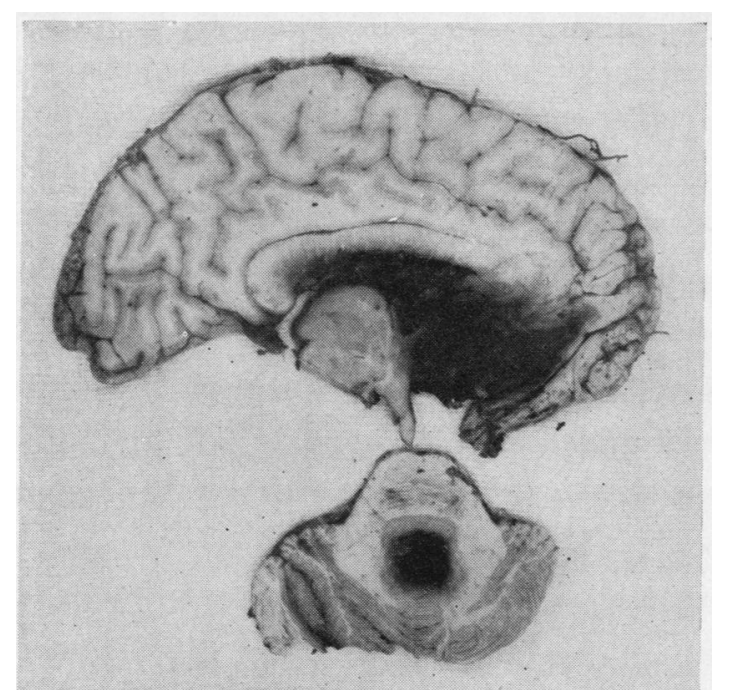

FIG. 3.-A massive collection of blood burrowing into sulci on the inferomedial surface of the frontal lobe and causing intraventricular haemorrhage by tearing the rostrum of the corpus callosum. unconscious a few days before death. Further, small areas of infarction of some standing were present in the frontal lobes, for new blood vessel formation and astrocytic proliferation had occurred and the peripheral parts of the blood clot itself were being organized.

A second case presented almost identical pathological findings.

In the third case a blood clot, $3 \mathrm{~cm}$. across, separated the frontal lobes, opening up the sulci on the medial surface on the left side, with numerous narrow tracks of blood into the hemisphere. This, like Robertson's (1949) similar case, was accompanied by infarction over the distribution of both anterior cerebral arteries. The fourth case presented a similar blood clot between the frontal lobes with necrosis limited to the frontal lobes in the immediate vicinity of the clot. In the fifth case much blood was present in the basal cisterns, but the Sylvian fissures and parieto-temporal sulci had been greatly widened, and blood had extended into the olfactory sulcus from below, producing what at first sight appeared to be an intracerebral haemorrhage (Fig. 4). The triangular-shaped blood clot was, however, almost completely surrounded by grey matter and was confined to the sulcus except at its posterior limit, where it had slightly extended into the brain. Infarction of the insular cortex and putamen was present. The sixth case showed an extension of blood over the superior surface of the infarcted and collapsed corpus callosum (Fig. 5), and there was massive bilateral infarction of anterior cerebral artery distribution. An attempt to clip the aneurysm, five days before death, was not responsible for the findings, for the histology was that of infarction of several weeks' standing and the diagnosis of frontai lobe infarction was suggested before operation. Similar cases have been seen by Robertson (1949) and Maloney (1958).

In two other instances the olfactory sulcus has been markedly distended by blood, and clot more. than $1 \mathrm{~cm}$. wide has been seen in the Sylvian fissure also twice. In a further case, from rupture of an internal carotid aneurysm, blood had: tracked into, and greatly distended, many sulci on the inferomedial surface of the hemisphere on the side of the aneurysm, with little trace of bleeding over the gyri.

Intracerebral and Intraventricular Haemorrhage.-Bleeding into the brain occurred in 17 of the 32 cases $(53 \%)$, and in 12 of these massive bleeding reached the ventricles. The route to the ventricles was through the rostrum of the corpus 


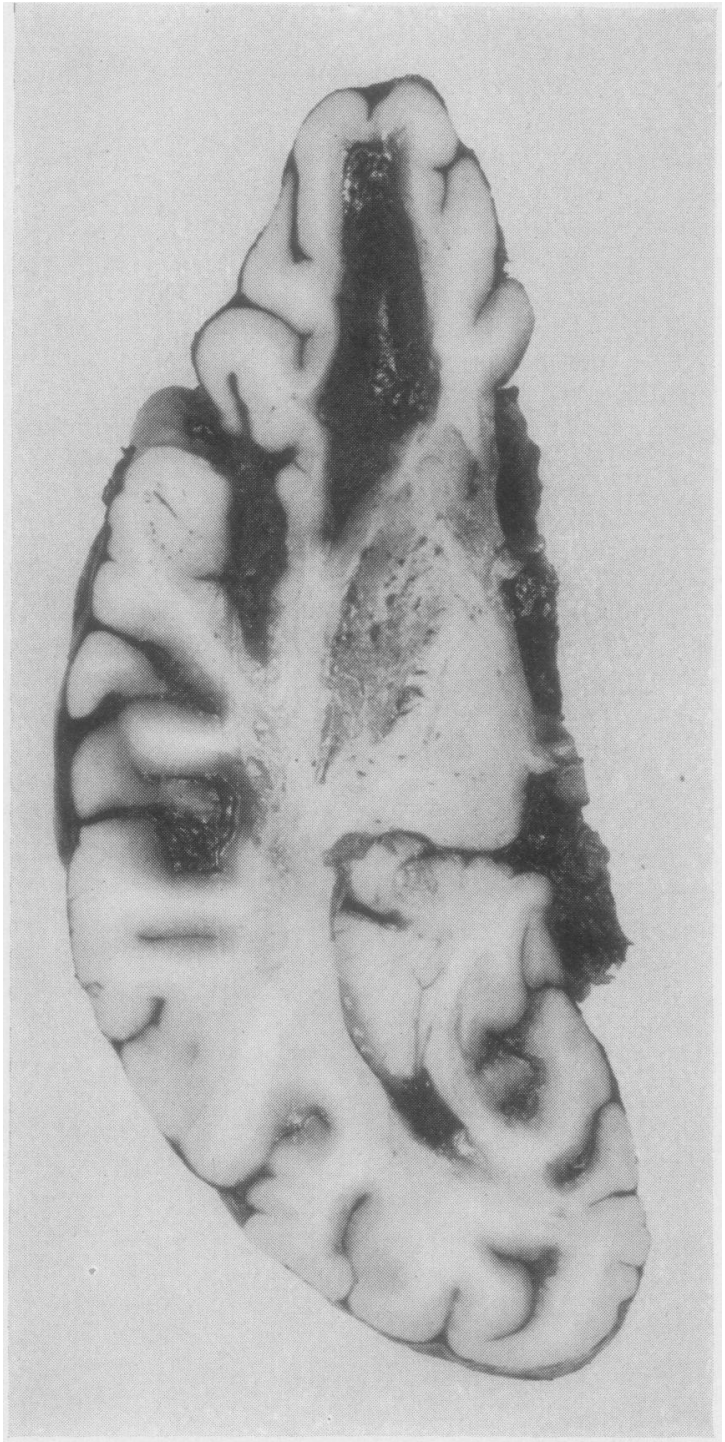

Fig. 4.-A massive collection of blood in a greatly widened olfactory sulcus; much blood in Sylvian fissure and depths of neighbouring sulci. Infarction of putamen.

callosum in seven, the insular cortex in one, and the frontal lobe to the anterior horn in four. In three cases where the rostrum was torn, haematomata were present in the frontal lobe. Of the five instances of intracerebral haematomata without rupture into the ventricles, three were into the basal ganglia through the insular cortex, one into the temporal lobe, and one into the frontal lobe. An important point in relation to these 17 instances is that in only six was the aneurysm adherent to or embedded in cerebral tissue, and the bleeding into the brain was therefore not direct.

A little blood was present in the ventricles in a further six instances; in two of these it had reached the anterior horn of the lateral ventricle via narrow haemorrhagic tracks through the cerebral substance, but in four blood had apparently reached the ventricles through the foramina of the fourth ventricle.

The percentage of intracerebral haemorrhage in this series is somewhat lower than the 60-75 which is usually recorded, but it would have been considerably higher $(68 \%)$ if subarachnoid haematomata had not been separated from intracerebral bleeding. One must also distinguish massive intracerebral bleeding from the linear haemorrhages which are common in the neighbourhood of the ruptured aneurysm. These latter, histologically, are perivascular and often run at right angles to the cerebral surface, and when numerous they are usually accompanied by parenchymatous degeneration. Their appearances strongly suggest that they result from blood tracking along the Virchow-Robin spaces into the cerebral tissue itself, forming linear intracerebral haemorrhages. It is clearly possible that coalescence of numerous haemorrhages produced in this fashion may result in a large haematoma.

Massive bleeding into the ventricles usually causes death in less than six hours (Bebin and Currier, 1957). Blood cannot escape rapidly from the ventricles (Symonds, 1924), and great dilatation commonly follows intraventricular bleeding. The fourth ventricle is often dilated to several times its normal size, with consequent distortion and pressure on pontine, medullary, and cerebellar structures. Haemorrhages, oedema, and occasionally actual infarction in the roof of the fourth ventricle result, and, less frequently, lesions in the medulla and pons. Subependymal haemorrhages also occur in the lateral ventricles, and less commonly in the peri-aqueductal tissues and anterior hypothalamus. Hypothalamic haemorrhages and ne:rosis are common with rupture of the rostrum, and in this series have been seen nine times; in eight the lesions were in the anterior hypothalamus. In six instances this hypothalamic damage was associated with massive intraventricular haemorrhage; in the other three instances the ruptured aneurysm was twice situated on the anterior cerebral and once on the internal carotid artery. 


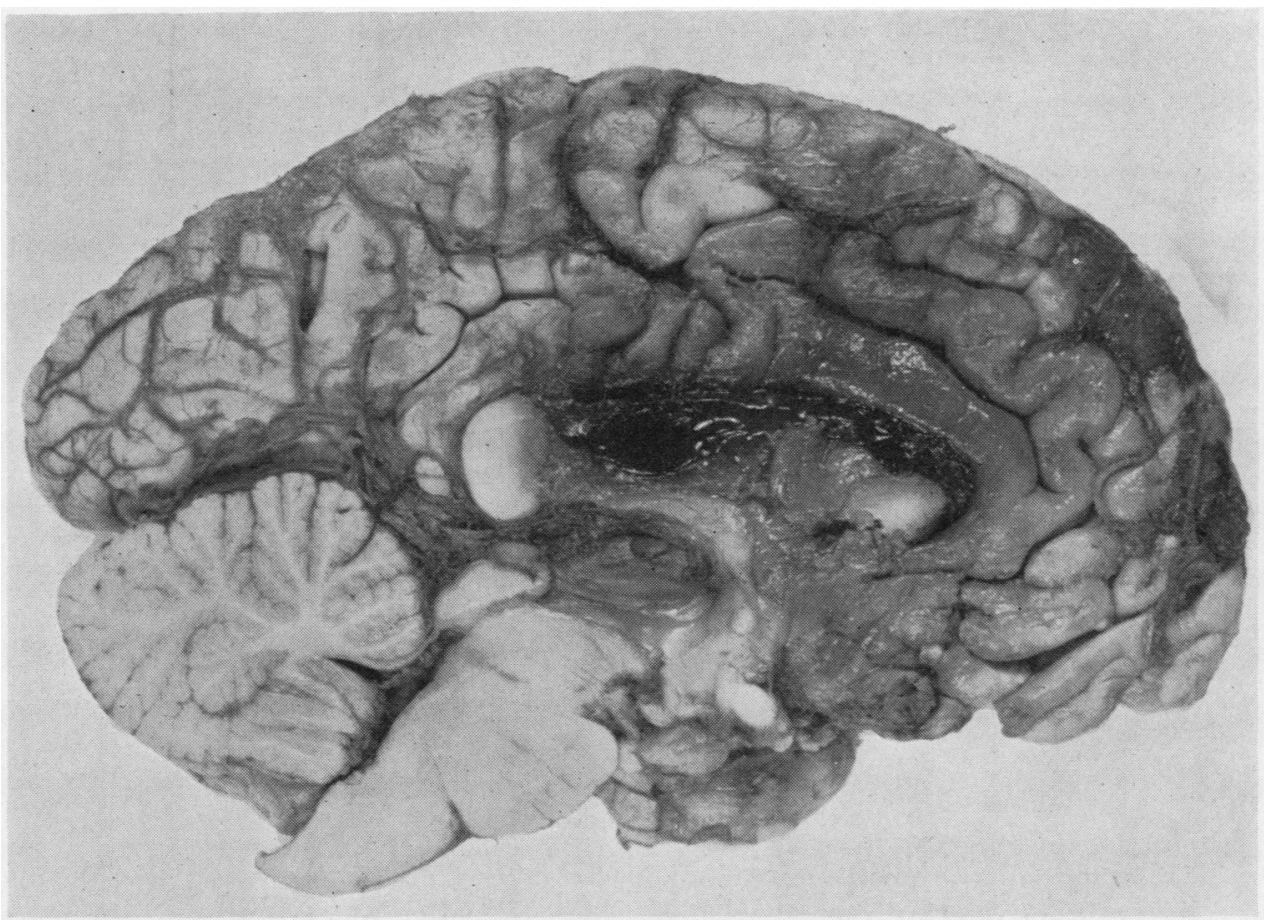

FIG. 5.-Blood clot lying over infarcted corpus callosum. Infarction of medial surface of anterior half of hemisphere.

Haemorrhages or degeneration, separately, or together, in the pons or cerebral peduncles, have been seen nine times, and in all but one instance, where infarction in the distribution of the posterior cerebral artery was present, they were considered to be due to increased supratentorial pressure. In four instances they accompanied massive intraventricular haemorrhage, in three subarachnoid bleeding without intracerebral bleeding, and in one massive infarction with great swelling of a hemisphere.

Cerebral Infarction. - Localized degeneration and gliosis up to $1 \mathrm{~cm}$. in depth is almost invariable around aneurysms embedded in cerebral tissue. This lesion may be asymptomatic and found in people who die from a single rapidly fatal haemorrhage. In addition, ischaemic lesions, varying from microscopic foci to massive infarctions, are very common. In this series 25 of the 32 brains examined $(78 \%)$ showed ischaemic lesions, and in $13(40 \%)$ these were massive. The smaller lesions are often multiple, and cortical foci up to $1 \mathrm{~cm}$. across may be separated by areas of normal brain, and in the fixed specimen appear as pale, irregularly round areas in the cortex, to which, if numerous, they impart a " moth-eaten" appearance. Similar, scattered foci are infrequently seen in the deep white matter, and occasionally, as reported by Falconer (1954), they occur in the depths of the sulci. If the foci are few, they are usually in the immediate neighbourhood of the ruptured aneurysm. They are particularly common on the medial surface of the hemisphere with ruptured aneurysms on or near the anterior communicating vessels. Four instances of such lesions have been seen in this series. In ruptured middle cerebral aneurysms similar foci occur on the insular cortex but also in the parietal or temporal lobe well away from the source of bleeding. With ruptured internal carotid aneurysms ischaemic foci may occur in the territory of both the middle and anterior cerebral arteries.

Of the 13 major infarctions, four involved the cortical territory of the anterior cerebral artery, on three occasions bilaterally. In two examples of bilateral infarction, the aneurysm was on the anterior communicating artery; in the third it was on the internal carotid, and patchy infarction of middle cerebral artery distribution was also present. In these three cases the cortex and underlying white matter was to a large extent destroyed in all sections from the medial surface of both hemispheres as far as the occipito-parietal 
sulcus and of the corpus callosum. In the fourth case the aneurysm was on the anterior communicating artery and one frontal lobe showed massive infarction, the other only scattered ischaemic foci. In three of the above infarction was limited to the cortical supply of the artery, but in one areas of infarction in the head of the caudate nucleus and anterior limb of the internal capsule indicated involvement of central branches. In two of the above four instances the infarction was associated with subarachnoid haematomata, once between the anterior frontal lobes and once over the corpus callosum. In the other two, massive intraventricular haemorrhage had occurred through rupture of the rostrum, and in one of these a large haematoma filled the olfactory sulcus. Since massive intraventricular rupture is probably rapidly fatal, it is likely in these cases that considerable haemorrhage anterior to the rostrum was present for some time before its rupture.

In eight cases there was infarction of a large area of brain supplied by the middle cerebral artery. In three the aneurysm was on the middle cerebral artery on the same side as the infarction and in three it was on the internal carotid on that side. In two it was on the anterior communicating artery ; in one of these a haematoma occupied the frontal lobe. In both cases the only infarction was in the territory of the middle cerebral artery. In three of the eight cases almost all the cortex supplied by the middle cerebral artery was infarcted, and in two of these the territory of the posterior cerebral artery was also involved (Fig. 6). In the other five cases a considerable area of cortex supplied by the middle cerebral artery was infarcted; in two the insular was most severely involved. A considerable depth of white matter was involved in these infarctions, usually reaching the lateral ventricle; in two instances foci of softening throughout the centrum semiovale were more obvious to the naked eye than cortical lesions, though the latter were extensive microscopically. Foci of infarction were found in the basal ganglia only once, indicating that the central branches of the middle cerebral artery usually escape involvement. In four of these eight instances a layer of blood more than $1 \mathrm{~cm}$. thick was present in the Sylvian fissure. Two of the remaining four had an intracerebral haematoma, one in the frontal lobe, the other in the anterior temporal lobe.

One instance of infarction of the entire territory of the posterior cerebral artery alone was seen, this associated with a ruptured aneurysm

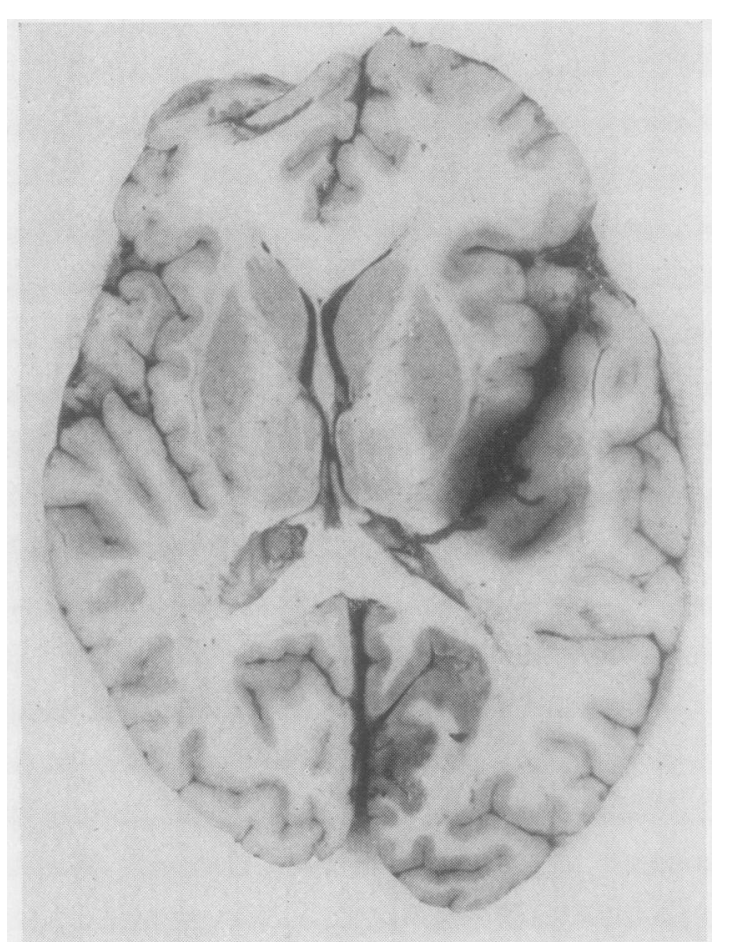

FIG. 6.-Infarction of middle and posterior cerebral artery territory from rupture of an aneurysm on the middle cerebral artery.

involving the basilar and posterior cerebral arteries. Only moderate bleeding around the ruptured aneurysm was present. The two instances of posterior cerebral artery infarction associated with middle cerebral artery lesions were probably the result of supratentorial pressure from the bleeding and from swelling of the hemisphere from the middle cerebral lesion. Feigin (1955) reported two similar cases.

In most instances the age of the infarction, based on histological appearances, corresponded approximately to the time of a major clinical episode. Two patients dying less than 24 hours after a single haemorrhage showed early, massive infarction, with patchy coagulative necrosis, numerous haemorrhages and oedema, and shrinkage of neurones in the involved tissue. A subject dying after three days showed great oedema of both frontal lobes with disintegration of the majority of neurones, myelin sheaths, and oligodendroglia. One man living for seven days showed great swelling of both frontal lobes with much neuronal destruction, increase of cellularity, new blood vessel formation, and well-marked softening of the white matter. Patients surviving longer than this showed progressively more 
advanced softening of the white matter with distinct astrocytic proliferation after three weeks.

Of the 13 instances of major infarction, eight had only one episode of bleeding, but in the other five the course suggested recurrent haemorrhages, and in two of these the histology and symptomatology suggested that the infarction occurred with the second and not the initial haemorrhage. In a further case there were infarctions of obviously different ages, one related to the original bleeding six weeks before death, and the other to the final bleeding a few hours before death.

There was no evidence, from the clinical course of the patients in this series, that investigation or operation contributed to the development of infarction. Apart from the two cases eliminated because of surgery, two others underwent major operative procedures. In one, no infarction was present; in the other, frontal lobe infarction was correctly diagnosed before the operation. Two cases had exploratory burr holes; one had an infarction, but the operation was performed immediately before death and could not have produced the ischaemic lesion.

Eighteen cases received no treatment or only antibiotics. In this group there were four major infarctions. In the treated group five showed clinical evidence of a cerebral lesion which proved to be an infarction, before treatment or investigation was begun. Therefore, in nine cases a major ischaemic lesion developed independently of treatment.

Ten of the treated cases were investigated by angiography without adverse effects. Four cases with major infarctions received, for the treatment of decerebrate rigidity or multiple convulsions, medication capable of producing hypotension, and in one of these a severe episode of hypotension was recorded. Possibly, therefore, the ischaemic lesion in these patients was influenced by medication. There is no way of determining this; all four were gravely ill when treatment was begun, and possibly already had ischaemic lesions. But, clearly, hypotension in a patient with cerebral ischaemia is likely to increase the severity of the lesion.

Degeneration of Granular Layer of Cerebellum. - Four instances of degeneration of the granular layer of the cerebellum were seen. The histological features in these cases were very similar to those described by Leigh and Meyer (1949) in association with visceral carcinomata and hypoglycaemic coma; they also mentioned its occurrence in a case of subarachnoid haemorrhage. The lesions varied from necrosis of all the nucleated elements in the granular layer to patchy severe destruction of the granular layer in which Golgi type 2 cells were preserved. In one case severe destruction of the granular layer was present in all sections; the other three showed patchy destruction, one in all areas examined, the other two mainly in the dorsomedial folia. The Bergmann glia appeared unaffected, and the Purkinje cells were not reduced in number though, in one case, some showed acute chromatolysis. There was no evidence of reaction to the necrotic foci, and degeneration of myelin and neurofibrils, glial proliferation, or excess of lipoids could not be demonstrated. No changes were found in the dentate nucleus or the inferior olives.

The ruptured aneurysm in three of these cases was on the internal carotid artery and in the fourth on the anterior communicating artery. The bleeding was massive in three and slight in the fourth.

The lack of reaction to the degeneration suggests it was a terminal event. All four patients had an episode of subarachnoid bleeding three to seven weeks before death, with partial or complete recovery. All four died suddenly from a further bleeding at the end of this period of convalescence. Three had been in hospital after the original bleeding, and no evidence of cerebellar abnormality was noted.

\section{Discussion}

A variety of pathological lesions follows rupture of an intracranial aneurysm. With massive bleeding, death may occur quickly, and little be found beyond blood in the subarachnoid space or brain. In other cases bleeding leads to the development of subarachnoid or intracerebral haematomata which, acting as space-occupying lesions, result in temporal herniation, lesions in the brain-stem, and occasionally ischaemia of the occipital and temporal lobes and posterior thalamus. All these lesions have been seen in this series, and massive infarction with brain swelling can produce them. Ruptured anterior cerebral, anterior communicating, and middle cerebral aneurysms are particularly likely to produce haematomata between the frontal lobes or in the Sylvian fissure. From internal carotid aneurysms, blood may track into the Sylvian fissures, and from any aneurysm groups of sulci may be 
opened out by tracking blood. Blood may also spread down the Virchow-Robin spaces into the brain, producing perivascular haemorrhages which often extend into the white matter. When the brain is sufficiently stretched by a localized collection intracerebral bleeding occurs. This process is probably more common than the rupture directly into the cerebral tissue of an aneurysm which has become embedded in, or adherent to, the brain. Adhesions in the subarachnoid space, resulting from previous haemorrhage, would further hinder the escape of blood from sulci or fissures and increase the likelihood of intracranial rupture; the delicate rostrum of the corpus callosum is especially liable to be torn by a neighbouring haematoma.

With rupture of any aneurysm, ischaemic damage to some portion of the brain is highly likely and massive infarction is not uncommon.

In this series no instance of a thrombosed major artery has been seen. Embolism from the aneurysmal sac is an equally unlikely cause of the infarction, for many involve the entire territory of an artery and the clot would clearly have to lodge in the main stem of the artery where its detection would be easy. Small peripheral emboli could account for scattered infarctions, but in many hundreds of sections examined an occluded cortical artery has been -seen only once.

Three other possibilities exist to account for ischaemic lesions. First, localized collections of blood could, by stretching or kinking, greatly narrow the vessels (Johnson, Potter, and Reid, 1958). Second, a large mass of blood stretching a major vessel may tear small branches passing from the parent vessel to the brain. This would deprive small areas of brain of their blood supply and provide further sources of bleeding into the subarachnoid space or brain. Either or both of these mechanisms might account for scattered as well as major infarctions, and could be responsible for ischaemic lesions even when no localized collections are found at necropsy; stretching of vessels by a haematoma may lead to irreversible ischaemic damage, but if the blood later escapes into the generalized subarachnoid space or ventricles, no local collection will be found at necropsy.

The third possibility is that spasm may cause the ischaemic lesions. The radiological evidence that spasm occurs after subarachnoid haemorrhage is strong (Ecker and Riemenschneider, 1951; Norlén and Olivecrona, 1953). In peripheral arteries, stretshing is a powerful stimulus to spasm (Simmons, 1956), but the same cannot be said with certainty of the cerebral vessels. Localized blanching of the cerebral vessels may follow the production of brain swelling from intracerebral injection of saline (Wolff, 1936), and Byrom (1954) attributed focal spasm during experimental window insertion to stretching of the affected vessels. In the latter's experiments on rats local pressure on arteries did not result in arterial contraction, though rubbing vessels (Florey, 1925) produced spasm which varied with the animal used. The latter also observed spasm on either side of a point of rupture, though it was insufficient to stop bleeding entirely.

Therefore, three mechanisms exist which might lead to ischaemic lesions; these are stretching or kinking of vessels, tearing of small vessels, and spasm possibly induced by stretching. Sufficient evidence to incriminate one or more of these mechanisms is not at present available, but all of them might be produced by haematomata, and these are demonstrable in many instances.

When intraventricular bleeding occurs slowly the blood may, presumably, escape from the ventricles sufficiently quickly to prevent their gross distension. Massive bleeding with great dilatation of the ventricles is, however, common and clearly the rate of entry of blood into the ventricles is then greater than its speed of exit through the foramina. The original bleeding need not be rapid for this to occur. It is more likely that rapidly fatal intraventricular haemorrhage results from rupture of an intracerebral or subarachnoid haematoma into the ventricular system, the haematoma often having collected slowly. Obstruction to the escape of blood from the ventricles can occur from compression of the aqueduct after temporal herniation (Smyth and Henderson, 1938), but no instance of this has been noted in this series.

No explanation can be offered for the granulecell degeneration of the cerebellum, and it would appear to be a terminal phenomenon in the cases reported here.

Patients who survive rupture of an intracranial aneurysm without sequelae presumably escape major infarction and large intracerebral or subarachnoid haematomas. When recovery is complete the bleeding will have been either small or from a site where blood could freely escape into the generalized subarachnoid space. But in Walton's series (1956), only about one third were so fortunate, and the remainder had residual abnormalities. The cerebral lesions in these cases 
probably only differed in degree from those in the fatal cases. Intracerebral and subarachnoid haematomas will be largely absorbed with time, and ischaemia of varying degree probably accounts for most of the disabilities, though only a careful study of such cases would settle this point.

It may be inappropriate to discuss treatment on the basis of the findings in these fatal cases, but two points stand out. Draining the spinal subarachnoid space can only be of value when the blood has ready access from the bleeding site to the basal cisterns. When haematomata have formed, draining the spinal theca will be futile and dangerous. Secondly, some cases have subarachnoid or intracerebral collections which require immediate surgery. Attempts to deal with the aneurysm within a few hours of haemorrhage have frequently had dire results, but there is little to be lost in attempting simple drainage of entrapped collections through burr holes in patients who otherwise appear certain to die. The knowledge that these large collections are usually in or between the frontal lobes, or in the Sylvian fissure or temporal lobes, should make attempted evacuation a reasonable possibility in patients otherwise considered hopeless. The surgical attack on the actual ruptured vessel could be considered if improvement followed direct drainage of the blood.

\section{Summary}

The findings in the brain in 32 fatal cases of ruptured intracranial aneurysm are reported.

Blood tends to collect between the frontal lobes, in the Sylvian fissure, and in the depths of sulci, and in these positions may form large haematomata. These may cause death by acting as space-occupying lesions and may lead to intracerebral and intraventricular haemorrhage. They are probably of importance in the development of infarction.

Ischaemic cerebral lesions are very common and massive infarction was found in $40 \%$ in this series. Ischaemic lesions probably produce most of the residual disabilities in non-fatal subarachnoid haemorrhage. The possible mechanisms of production of ischaemic lesions are discussed.

I wish to thank my colleagues in the laboratory, particularly Dr. S. M. Bell and Dr. B. J. Smith, for their help in collecting this material; my clinical colleagues, particularly Mr. L. P. Lassman, for permission to peruse their records; Dr. A. F. J. Maloney for most valuable comments on the histological findings; and Mr. A. Hall, Mr. E. Manns, and Miss G. Hunt for technical assistance.

\section{REFERENCES}

Beadles, C. F. (1907). Brain, 30, 285.

Bebin, J., and Currier, R. D. (1957). A.M.A. Arch. intern. Med., 99 771 .

Bigelow, N. H. (1955). A.M.A. Arch. Neurol. Psychiat., 73, 76.

Byrom, F. B. (1954). Lancet, 2, 201.

Ecker, A., and Riemenschneider, P. A. (1951). J. Neurosurg., 8, 660.

Falconer, M. A. (1954). Proc. roy. Soc. Med., 47, 693.

Fearnsides, E. G. (1916). Brain, 39, 224.

Feigin, I. (1955). A.M.A. Arch. Neurol. Psychiat., 73, 463.

Florey, H. (1925). Brain, 48, 43.

Johnson, R. J., Potter, J. M., and Reid, R. G. (1958). J. Neurol. Neurosurg. Psychiat., $21,68$.

Leigh, A. D., and Meyer, A. (1949). Ibid., 12, 287.

Maloney, A. F. J. (1958). Personal communication.

Norlén, G., and Olivecrona, H. (1953). J. Neurosurg., 10, 404.

Richardson, J. C., and Hyland, H. H. (1941). Medicine (Baltimore), $20,1$.

Robertson, E. G. (1949). Brain, 72, 150.

Simmons, E. H. (1956). A.M.A. Arch. Surg., 73, 625.

Smyth, G. E., and Henderson, W. R. (1938). J. Neurol. Psychiat., n.s., 1, 226.

Symonds, C. P. (1924). Quart. J. Med., 18, 93.

Walton, J. N. (1956). Subarachnoid Haemorrhage. Livingstone, Edinburgh and London.

Wilson, G., Riggs, H. E., and Rupp, C. (1954). J. Neurosurg., 11, 128. Wolff, H. G. (1936). Physiol. Rev., 16, 545. 